\title{
Efficient capacity-based antenna selection for MIMO systems
}

Michael A. Jensen

jensen@byu.edu

Matthew L. Morris

Follow this and additional works at: https://scholarsarchive.byu.edu/facpub

Part of the Electrical and Computer Engineering Commons

\section{Original Publication Citation}

Jensen, M. A., and M. L. Morris. "Efficient Capacity-Based Antenna Selection for MIMO Systems." Vehicular Technology, IEEE Transactions on 54.1 (25): 11-6

\section{BYU ScholarsArchive Citation}

Jensen, Michael A. and Morris, Matthew L., "Efficient capacity-based antenna selection for MIMO systems" (2005). Faculty Publications. 399.

https://scholarsarchive.byu.edu/facpub/399 


\title{
Efficient Capacity-Based Antenna Selection for MIMO Systems
}

\author{
Michael A. Jensen, Senior Member, IEEE, and Matthew L. Morris
}

\begin{abstract}
The achieved capacity of the multiple-input-multiple-output wireless channel is typically dependent on the array configurations at the transmitter and receiver. Maximizing system capacity or throughput therefore requires that the arrays adapt to changing channel conditions, which may be accomplished by selecting an appropriate subset of available antenna elements for connection to the electronic transmit and receive modules. This paper presents algorithms, derived using relatively straightforward information theoretic considerations, for efficiently and effectively selecting the antenna elements. Computational examples using a realistic channel model for indoor environments illustrate the performance of the techniques.
\end{abstract}

Index Terms-Antenna arrays, information theory, multipleinput-multiple-output (MIMO) systems.

\section{INTRODUCTION}

$\mathbf{M}$ ULTIPLE-INPUT-MULTIPLE-OUTPUT (MIMO) wireless systems have demonstrated the potential for increased capacity in rich multipath environments [1]-[3]. In traditional studies of MIMO systems, the communication capacity is computed from the transfer matrix defining the response from each transmit to each receive antenna. However, this capacity depends on the antenna configuration [4]; therefore, maximizing the system throughput may require that this configuration adapt to changing propagation conditions. One mechanism for accomplishing this adaptation is to fabricate large arrays and use switching networks to dynamically connect different subsets of the elements to a smaller number of transmit and receive modules [5], [6]. To make this approach practical, however, efficient and effective methods for choosing the appropriate antenna element subset are required.

Antenna selection for MIMO systems has been considered for several scenarios. For example, recent studies reveal how antenna selection can increase capacity [7] or received signal-tonoise ratio (SNR) [8] and decrease symbol error rate [9] of MIMO systems. Further, [10] demonstrates substantially improved symbol error rates when using antenna selection in conjunction with simple linear receiver topologies. However, each of these studies examines antenna selection only at one end of the link and uses an exhaustive search to identify the optimal element set, an approach that becomes prohibitive for large array

Manuscript received March 4, 2003; revised February 12, 2004 and September 8, 2004. This work was supported by the National Science Foundation under Information Technology Research Grant CCR-0313056. The review for this paper was coordinated by Dr. A. Annamalai.

The authors are with the Department of Electrical and Computer Engineering, Brigham Young University, Provo, UT 84602 USA (e-mail: jensen@ee.byu.edu).

Digital Object Identifier 10.1109/TVT.2004.838886 sizes. A comprehensive look at MIMO antenna selection based upon minimizing probability of error while maximizing SNR is provided in [11]. However, this approach again utilizes an exhaustive search for antenna selection. Alternately, [12] proposes selecting transmit antennas based on the power computed from the water-filling capacity solution, an approach that is compared to the schemes derived here. Finally, [13] presents a suboptimal yet efficient iterative procedure for eliminating the antennas that contribute least to the capacity.

In this paper, we present alternate suboptimal yet efficient antenna-selection algorithms, suitable for application to large antenna arrays, based upon metrics derived from mutual information (MI) considerations. It is shown that, with little additional computational overhead, antennas obtained using these algorithms outperform those selected based on power alone. Computational results obtained using realistic channel models reveal the excellent performance of the techniques despite their computational simplicity.

\section{MIMO ANTENNA-SELECTION FRAMEWORK}

The algorithms developed in this paper are generally based on metrics related to the MI between the transmitted and received data symbols. Therefore, it is useful to begin by defining the communication model and notation assumed in the analysis and by providing a mathematical foundation for the proposed antenna-selection approaches. In the following derivation, boldface uppercase and lowercase letters will be used to represent matrices and vectors, respectively, with $h_{i}$ representing the $i$ th element of the vector $\mathbf{h}$ and $H_{i j}$ representing the element occupying the $i$ th row and $j$ th column of the matrix $\mathbf{H}$.

\section{A. MIMO System Capacity}

Consider a narrow-band wireless system that communicates over a general multipath channel using $N_{T}$ and $N_{R}$ antennas at the transmitter and receiver, respectively. In general, if the $N_{T} \times 1$ vector of complex baseband transmit symbols is denoted as $\mathbf{X}$, where $x_{i}$ is the symbol transmitted from the $i$ th antenna, then the $N_{R} \times 1$ vector of received symbols can be written as

$$
\mathbf{y}=\mathbf{H x}+\boldsymbol{\eta}=\mathbf{s}+\boldsymbol{\eta}
$$

where $\mathbf{H}$ is the $N_{R} \times N_{T}$ transfer matrix and $\boldsymbol{\eta}$ is a $N_{R} \times 1$ vector representing noise or measurement error. For the remainder of this paper, we will assume zero-mean complex Gaussian noise with covariance $\mathrm{E}\left\{\boldsymbol{\eta} \boldsymbol{\eta}^{H}\right\}=\sigma_{\eta}^{2} \mathbf{I}$, where $\mathbf{I}$ is the identity matrix, $\mathrm{E}\{\cdot\}$ denotes an expectation, and $\{\cdot\}^{H}$ is the Hermitian operator. 
Given the representation in (1), the capacity of the MIMO system can be computed using the water-filling solution [3], [14]. This computation yields two key items: 1) the capacity in bits per channel use (bits/s/Hz) and 2) the optimal transmit covariance matrix $\mathbf{K}_{x x}=\mathrm{E}\left\{\mathbf{x x}^{H}\right\}$. We can also compute the covariance matrices $\mathbf{K}_{s s}=\mathrm{E}\left\{\mathbf{s s}^{H}\right\}=\mathbf{H} \mathbf{K}_{x x} \mathbf{H}^{H}$ and $\mathbf{K}_{y y}=$ $\mathrm{F}\left\{\mathbf{y} \mathbf{y}^{H}\right\}=\mathbf{K}_{s s}+\sigma_{\eta}^{2} \mathbf{I}$ that are useful in the MI metrics outlined later in this section. This computation assumes the system possesses perfect channel estimates, although a modified waterfilling solution can be used instead when this is not the case [15].

\section{B. Selection Algorithms}

The system of interest in this paper possesses a larger set of antennas than transmit or receive electronics. For example, recent research in reconfigurable antennas suggests the potential for fabricating large antenna arrays and using inexpensive highperformance switching networks to adaptively connect different subsets of the elements to the transmit and receive modules. What is lacking is a technique for determining which subset of the antennas should be selected. For this work, the optimal combination of transmit and receive subarrays is that which yields the highest system capacity.

The most straightforward approach for selecting the optimal subarray is to exhaustively search over all possible combinations. However, this search quickly becomes computationally prohibitive with increasing array size. For example, an exhaustive search to select four antennas from transmit and receive arrays with 16 elements each requires computation of the capacity over 3.3 million combinations. This computational burden motivates the development of alternate more efficient selection approaches.

The problem can be simplified if we utilize the basic information resulting from the capacity computation, specifically the transfer matrix $\mathbf{H}$ and the computed covariance matrices. Since the diagonal elements of these covariance matrices are proportional to the average power transmitted or received by the individual antenna elements, one simple approach would be to select those elements with the highest power, as suggested in [12]. While this can be effective, for densely packed arrays the signals on a cluster of closely spaced elements can all be characterized by high power, but possess similar information content. From the standpoint of capacity, it may be better to choose only one element from this cluster and other lower-power signals, which provide additional information. This fact will be demonstrated by the results in Section III.

Effective algorithms for antenna selection should, therefore, look at the entire covariance matrix rather than simply the diagonal elements. One way to use this additional information is to form decision metrics based on MI quantities in combination, possibly, with the signal power. It should be emphasized, however, that utilizing the covariance matrix for a large array to select a subarray will generally lead to suboptimal results. This can be explained by recognizing that, for a specific channel, the optimal transmit covariance for the subset may be quite different from the covariance for the entire array. The goal of these algorithms, therefore, is to achieve high performance with computational efficiency.
The proposed algorithms are iterative, meaning that, at each step, computations are performed to determine which of the remaining elements should be selected next. As such, we introduce the set $\mathcal{A}$ that contains the indexes of the antennas already selected in the iterative process. The transmit vector containing the subset of signals represented in $\mathcal{A}$ is denoted as $\mathbf{x}_{\mathcal{A}}$ and has covariance $\mathbf{K}_{x x, \mathcal{A A}}$ consisting of the rows and columns of $\mathbf{K}_{x x}$ associated with the indices in $\mathcal{A}$. This notation also applies to the receive array using the substitution $x \rightarrow s$. In describing the algorithms, it is assumed that $\mathcal{A}$ has been initialized to contain at least one index.

1) High Power and Low MI Within an Array: The first proposed metric for antenna selection involves choosing elements with high signal power, but where the MI between the signal (element) under investigation and the already selected signals is low. For the transmit array, we therefore need to first compute the MI between the signal $x_{i}$ on the $i$ th antenna and the vector of signals $\mathbf{x}_{\mathcal{A}}$ on the already selected antennas. This quantity is given as [16]

$$
I\left(x_{i}, \mathbf{x}_{\mathcal{A}}\right)=\mathcal{H}\left(x_{i}\right)-\mathcal{H}\left(x_{i} \mid \mathbf{x}_{\mathcal{A}}\right)
$$

where $\mathcal{H}(\cdot)$ represents the entropy. The variance of $x_{i}$ conditioned on $\mathbf{x}_{\mathcal{A}}$ can be expressed as [17]

$$
\operatorname{var}\left\{x_{i} \mid \mathbf{x}_{\mathcal{A}}\right\}=K_{x x, i i}-\mathbf{k}_{x x, i \mathcal{A}} \mathbf{K}_{x x, \mathcal{A} \mathcal{A}}^{-1} \mathbf{k}_{x x, \mathcal{A} i}
$$

where, consistent with our notational convention, $\mathbf{k}_{x x, i \mathcal{A}}$ and $\mathbf{k}_{x x, \mathcal{A} i}$ are row and column vectors, respectively, containing the elements identified by the indices in $\mathcal{A}$ of the $i$ th row and $i$ th column of $\mathbf{K}_{x x}$, respectively. The MI becomes

$$
I\left(x_{i}, \mathbf{x}_{\mathcal{A}}\right)=\log _{2} \frac{\left|K_{x x, i i}\right|}{\left|K_{x x, i i}-\mathbf{k}_{x x, i \mathcal{A}} \mathbf{K}_{x x, \mathcal{A}}^{-1} \mathbf{k}_{x x, \mathcal{A} i}\right|} .
$$

It will be convenient to make the relative weight of the MI on the same order of magnitude as the power $K_{x x, i i}$ of the signal $x_{i}$. Therefore, as a measure of MI, we will use the argument of the logarithm expressed as

$$
Q\left(x_{i}, \mathbf{x}_{\mathcal{A}}\right)=\frac{\left|K_{x x, i i}\right|}{\left|K_{x x, i i}-\mathbf{k}_{x x, i \mathcal{A}} \mathbf{K}_{x x, \mathcal{A} \mathcal{A}}^{-1} \mathbf{k}_{x x, \mathcal{A} i}\right|} .
$$

Note that the MI metric for signals on antennas in the receive array is given by (5) after making the substitution $x \rightarrow s$.

To generalize this result, let $\mathbf{K}$ represent the covariance matrix $\mathbf{K}_{x x}$ or $\mathbf{K}_{s s}$, depending on whether we are applying the algorithm for transmit or receive antenna selection, respectively. A signal that has high average power $K_{i i}$ but has low MI with the already selected signals will have a large value of the ratio of $K_{i i}$ to $Q$ in (5) or

$$
D_{i \mathcal{A}}=\left|K_{i i}-\mathbf{k}_{i \mathcal{A}} \mathbf{K}_{\mathcal{A} \mathcal{A}}^{-1} \mathbf{k}_{\mathcal{A} i}\right|
$$

Note that (6) is simply the variance of the signal on the $i$ th element conditioned on the signals on the already selected elements. If these selected signals are fixed and the signal on the element under investigation is highly correlated to the selected antennas, then this variance will be low, suggesting that the $i$ th 
antenna will provide little additional information beyond what can be obtained from the already selected antennas.

For the algorithm implementation, we initially select the element characterized by the highest average power so that $\mathcal{A}$ contains the index of this antenna. The metric in (6) is then computed for all $i, i \notin \mathcal{A}$ and the antenna producing the highest metric is selected. The set $\mathcal{A}$ is then augmented to include this index and the process is repeated until the desired number of antennas has been selected. This algorithm is applied to the transmit and receive arrays independently, resulting in low computational cost.

Other methodologies for using this metric can be constructed, such as rejecting the antennas offering the lowest values of the metric either all at once or using iterative algorithms. However, we have found that such variants generally do not perform as well as this simple selection approach and require significantly increased computational cost, since larger matrices are used in the matrix-vector products. Therefore, they will not be considered further.

2) High Transmit/Receive MI: The second proposed metric for antenna selection involves choosing elements that maximize the MI between the signals on the transmit and receive arrays. To enable this approach for receive-antenna selection, we will compute the MI between a partition $\mathbf{y}_{\mathcal{A}}$ of the receive vector $\mathbf{y}$ and the transmit vector $\mathbf{x}$. For the signal model in (1), this MI expression can be written as

$$
I\left(\mathbf{y}_{\mathcal{A}}, \mathbf{x}\right)=\log _{2} \frac{\left|\left(\mathbf{H K}_{x x} \mathbf{H}^{H}-\sigma_{\eta}^{2} \mathbf{I}\right)_{\mathcal{A A}}\right|}{\left|\sigma_{\eta}^{2} \mathbf{I}_{\mathcal{A A}}\right|}
$$

where $|\cdot|$ represents a determinant. Again, using the argument of the logarithm leads to

$$
Q\left(\mathbf{y}_{\mathcal{A}}, \mathbf{x}\right)=\frac{\left|\left(\mathbf{H} \mathbf{K}_{x x} \mathbf{H}^{H}-\sigma_{\eta}^{2} \mathbf{I}\right)_{\mathcal{A A}}\right|}{\left|\sigma_{\eta}^{2} \mathbf{I}_{\mathcal{A A}}\right|} .
$$

For transmit-antenna selection, we need to compute the MI between $\mathbf{y}$ and a partition $\mathbf{x}_{\mathcal{A}}$ of $\mathbf{x}$ or

$$
I\left(\mathbf{y}, \mathbf{x}_{\mathcal{A}}\right)=\mathcal{H}(\mathbf{y})-\mathcal{H}\left(\mathbf{y} \mid \mathbf{x}_{\mathcal{A}}\right)=\mathcal{H}\left(\mathbf{x}_{\mathcal{A}}\right)-\mathcal{H}\left(\mathbf{x}_{\mathcal{A}} \mid \mathbf{y}\right)
$$

where we have used the symmetry property of the information to arrive at the latter equality. This last form is more computationally efficient, since vectors in the first expression are larger than those in the second expression. However, we must compute the covariance

$$
\begin{aligned}
\mathbf{T}_{\mathcal{A A}} & =\operatorname{cov}\left\{\mathbf{x}_{\mathcal{A}} \mid \mathbf{y}\right\} \\
& =\left[\mathbf{K}_{x x}-\mathbf{K}_{x y} \mathbf{K}_{y y}^{-1} \mathbf{K}_{y x}\right]_{\mathcal{A A}} \\
& =\left[\mathbf{K}_{x x}-\mathbf{K}_{x x} \mathbf{H}^{H} \mathbf{K}_{y y}^{-1} \mathbf{H} \mathbf{K}_{x x}\right]_{\mathcal{A A}} .
\end{aligned}
$$

The argument of the logarithm in the MI expression will, therefore, become

$$
Q\left(\mathbf{y}, \mathbf{x}_{\mathcal{A}}\right)=\frac{\left|\mathbf{K}_{x x, \mathcal{A A}}\right|}{\left|\mathbf{T}_{\mathcal{A A}}\right|}
$$

In the selection algorithm using these quantities, we first let $\mathcal{B}_{i}$ represent the set of previously selected indexes $\mathcal{A}$ plus the index $i$, where $i \notin \mathcal{A}$. Initially, $\mathcal{B}_{i}$ contains only $i$. When selecting transmit or receive antennas, the value of $i$ that maximizes the value of $Q\left(\mathbf{y}, \mathbf{x}_{\mathcal{B}_{i}}\right)$ or $Q\left(\mathbf{y}_{\mathcal{B}_{i}}, \mathbf{x}\right)$, respectively, is selected and added to the set $\mathcal{A}$. This procedure is then repeated until the set $\mathcal{A}$ contains the desired number of antennas. In the examples shown later, this is implemented such that the transmit antennas are first chosen to maximize (in this iterative sense) $Q\left(\mathbf{y}, \mathbf{x}_{\mathcal{A}}\right)$. Then, the required covariance matrices are recomputed from the water-filling capacity formulation using the columns of $\mathbf{H}$ corresponding to the chosen transmit antennas. The receive antennas are then selected based on these updated covariance matrices. This approach yields substantially improved performance over simply independently maximizing $Q\left(\mathbf{y}_{\mathcal{A}}, \mathbf{x}\right)$ and $Q\left(\mathbf{y}, \mathbf{x}_{\mathcal{A}}\right)$.

3) Transfer Matrix Row/Column Selection: Instead of using the full-array covariance matrix, we can iteratively choose the rows and columns the transfer matrix $\mathbf{H}$ that offer the best incremental capacity. We select the first transmit antenna by computing the capacity for each column of $\mathbf{H}$ and choosing the column $i$ (whose index is placed in $\mathcal{A}$ ) that offers the largest capacity. We then compute the capacity for the matrix formed from the already selected column (in $\mathcal{A}$ ) combined with each remaining column individually. The column that yields the maximum capacity is then selected and its index is added to $\mathcal{A}$. This procedure is repeated until the desired number of antennas has been selected. An identical procedure can be applied to the rows of the new transfer matrix consisting of the chosen columns to perform receive-antenna selection.

\section{Algorithmic Computational Cost}

Before demonstrating the performance of the various antenna selection algorithms, it is worthwhile to compare their relative approximate computational costs. For discussion, we will assume that we are selecting $M$ out of $N$ antennas for both transmit and receive. The cost of the singular value decomposition (SVD) of an $n \times m$ matrix required for the water-filling capacity computation (and, therefore, covariance matrix construction) is $\mathcal{C}_{\mathrm{SVD}}=12 \mathrm{~nm}^{2}+9 \mathrm{~m}^{3}$ [18]. Therefore, for all methods that first require construction of the full covariance matrices (size $N \times N$ ) of the signals on all antennas, there is an initial cost of $\mathcal{O}\left(N^{3}\right)$, where $\mathcal{O}(\cdot)$ represents the order of the computation. We will use this notion of computational order throughout the remainder of this discussion.

1) Signal Power: Selecting elements based on the diagonal terms of the covariance matrix (signal power) only requires computation of the initial covariance matrices. Therefore, this approach has computational cost $\mathcal{O}\left(N^{3}\right)$.

2) High Power, Low MI Within an Array: In addition to the initial covariance matrix computation, at the $k$ th iteration of this approach $1 \leq k<M$, we have $N-k$ vector-matrix-vector multiplies each of cost $(k+1) k$ operations. If $M \ll N$, the cost for these operations roughly scales as $\mathcal{O}\left(N M^{2}\right)$. This cost must, therefore, be added to the $\mathcal{O}\left(N^{3}\right)$ computation of the initial 


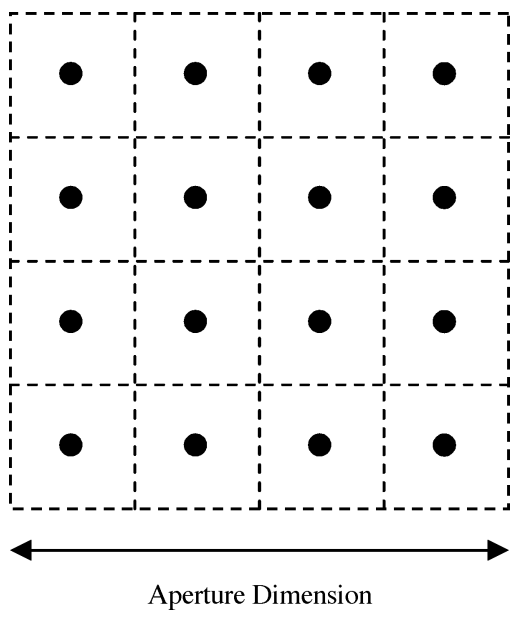

Fig. 1. Antenna aperture divided into a two-dimensional grid. The antenna elements are constrained to be placed at the centers of the grid cells (denoted by dots).

SVD. This incremental cost is relatively small for small values of $M$.

3) High Transmit/Receive MI: In addition to the initial covariance matrix computation, we must perform an initial $N \times N$ matrix multiply of cost $\mathcal{O}\left(N^{3}\right)$ as well as determinant computations in each iteration, the largest of which has cost $\mathcal{O}\left(M^{3}\right)$. If $M \ll N$, then the dominant incremental cost incurred stems from the matrix product.

4) Transfer Matrix Row/Column Selection: This algorithm does not use the initial SVD required for the covariance matrix construction, but does require computation of an SVD at each iteration. If we first select the columns of $\mathbf{H}$ (transmit antennas), then at the $k$ th iteration, $1 \leq k \leq M$, we compute $N-k+1$ SVDs of $N \times k$ matrices, each with a cost of $\mathcal{O}\left(N k^{2}\right)$. Since the largest $\mathrm{SVD}(N \times M$ matrix $)$ dominates the cost expression, for $M \ll N$ we observe a rough cost of $\mathcal{O}\left(N^{2} M^{2}\right)$. Once the transmit antennas have been selected, the SVD computations for the receive-antenna selection are of reduced size (largest matrix is $M \times M$ ) and, therefore, do not dominate the computation. More generally, the computational cost of this approach can be somewhat less or more than that of the other algorithms, depending on the ratio of $M / N$. Generally speaking, we have found that this algorithm executes faster than those requiring the initial SVD computation.

5) Exhaustive Search: Choosing $M$ out of $N$ antennas leads to $N ! / M !(N-M)$ ! combinations for each array. For each transmit-antenna combination, we must search over all combinations of the receive antennas, so that the total number of combinations is $[N ! / M !(N-M) !]^{2}$. The capacity of the $M \times M$ submatrix must then be computed for each scenario, leading to the potentially huge overall computational cost of $\mathcal{O}\left\{[N ! / M !(N-M) !]^{2} M^{3}\right\}$.

The conclusion of this analysis is that the algorithms all share roughly the same computational cost, although higher algorithmic complexity tends to lead to some additional burden. Naturally, the feasibility of implementing these algorithms in a real-time communication system will depend on the array sizes as well as the temporal channel variability that dictates the frequency of updating the selected antenna subset.
TABLE I

Capacity of the Best Array SElected From 100, 1000, and 5000 RANDOMLY GENERATED ARRAYS AS WELl AS THE CAPACITY OF THE Optimal ARRAy ObTaINED by AN EXHAUSTIVE SEARCH FOR THREE- AND FOUR-ElEMENT ARRAYS PlACED IN A NINE-ELEMENT GRID

\begin{tabular}{l|c|ccc}
\hline \# Elements & Exhaustive & \multicolumn{3}{|c}{ Random Arrays } \\
& Search & 100 & 1,000 & 5,000 \\
\hline 3 & 13.3 & 12.4 & 13.3 & 12.9 \\
4 & 15.8 & 14.7 & 15.4 & 15.7 \\
\hline
\end{tabular}

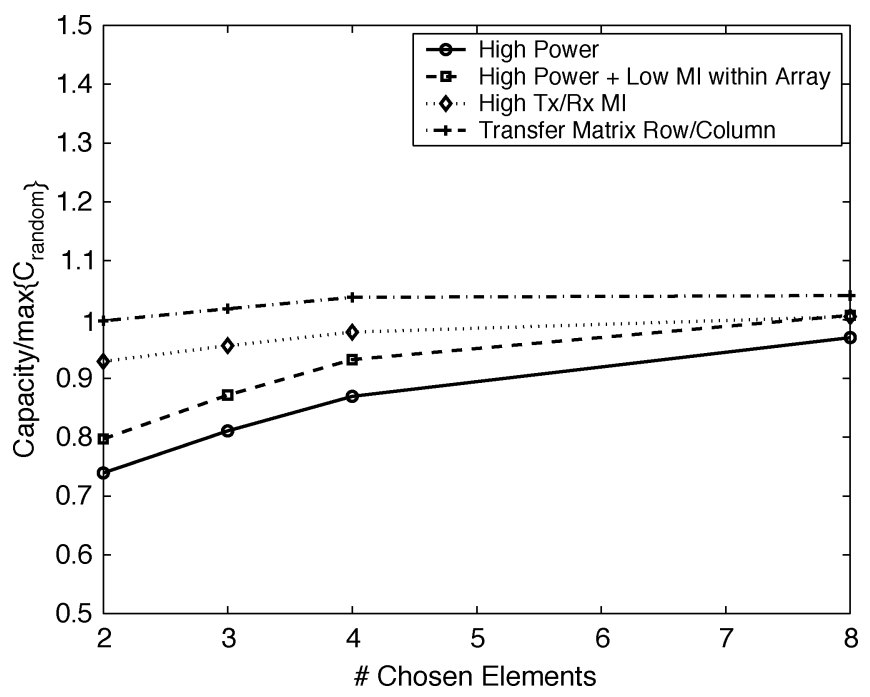

Fig. 2. Selected array capacity normalized by the maximum capacity from 5000 randomly generated arrays versus the number of selected antenna elements. Each point represents an average computed from 150 different channels. The $2 \lambda$ apertures are divided into two gridpoints per wavelength.

\section{COMPUTATIONAL EXAMPLES}

To illustrate the relative performance of the proposed algorithms, MIMO channels were created using the path-based Saleh-Valenzuela model extended with angle of departure/arrival information, referred to as the SVA model [19], [20]. Square transmit and receive apertures are subdivided into square sections and antenna elements with omnidirectional patterns in the horizontal plane are placed at the subdivision centers, as depicted in Fig. 1. For each channel, the transfer matrix $\mathbf{H}$ is created for the aperture under investigation and the relevant covariance matrices are computed using the water-filling capacity formulation [3], [14]. The single-input-single-output (SISO) SNR, as defined in [19], is set to $20 \mathrm{~dB}$ in each computation.

First, many channels were generated using the SVA model and 150 of these channels that offered a relatively uniform capacity distribution for $2 \lambda$ square transmit and receive apertures ( $\lambda$ is the free-space wavelength) were selected for the computations. For each channel realization, 5000 random arrays consisting of two, three, four, and eight elements and conforming to the array grid were generated and the capacity of each array was recorded. We will use the symbol $\mathrm{C}_{\text {random }}$ to denote this set of capacities for all array realizations of a given number of elements and for a given channel. Also, for each channel and array size, the capacity $\mathrm{C}_{\text {square }}$ of a "square" array placed around the grid perimeter was computed. For two- and three-element arrays, this "square" array consisted of either two elements at opposite corners of the square or the isosceles triangle formed by 


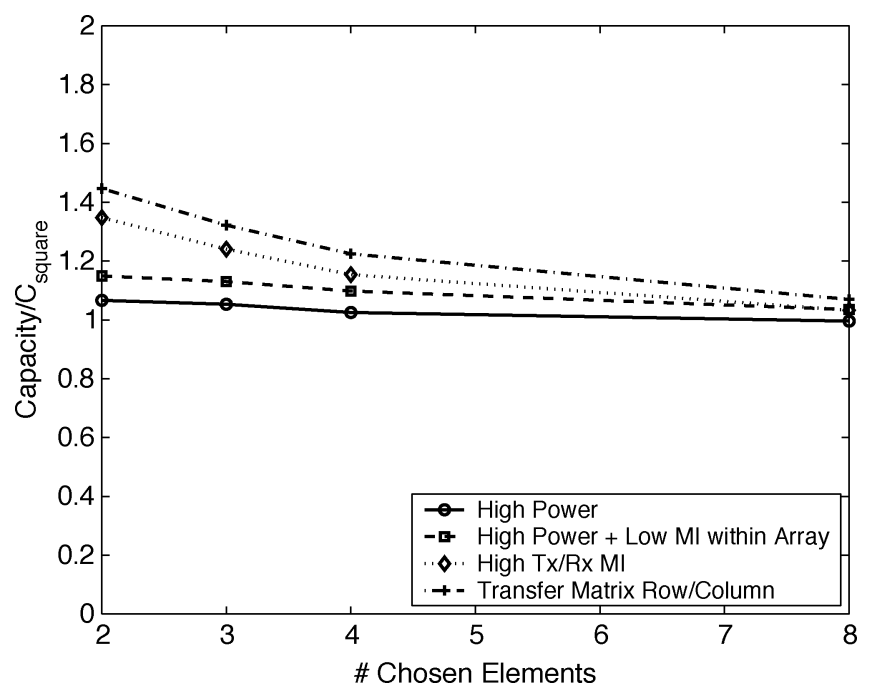

Fig. 3. Selected array capacity normalized by the capacity for the appropriate square array versus the number of selected antenna elements. Each point represents an average computed from 150 different channels. The $2 \lambda$ apertures are divided into two gridpoints per wavelength.

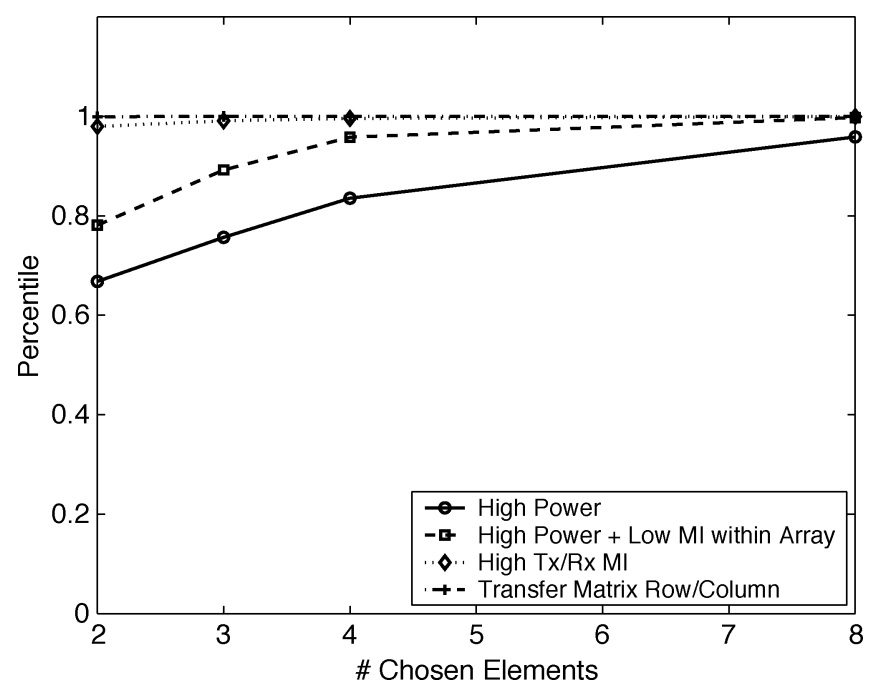

Fig. 4. Percentile performance of the selected array capacity relative to the capacity statistics obtained from 5000 randomly generated arrays. Each point represents an average computed from 150 different channels. The $2 \lambda$ apertures are divided into two gridpoints per wavelength.

using elements at two adjacent corners and the middle of the opposite side, respectively.

Because of the large array sizes considered here, an exhaustive search for the optimal antenna subset is impractical. Instead, the maximum capacity value obtained from the 5000 randomly generated arrays is used to approximate this optimal capacity. To assess the quality of this approximation, a specific channel realization was created and transmit and receive arrays consisting of three or four elements constrained to a $3 \times 3$ grid on $2 \lambda$ square apertures were formed. An exhaustive search was used to identify the optimal array configurations, after which the best array out of 100, 1000, and 5000 random realizations was chosen. Table I shows the capacities obtained by these methods. As can be seen, the random search in some cases finds the optimal array and generally provides a reasonable estimate of the optimal performance. Naturally, for larger grids, the ability of the random

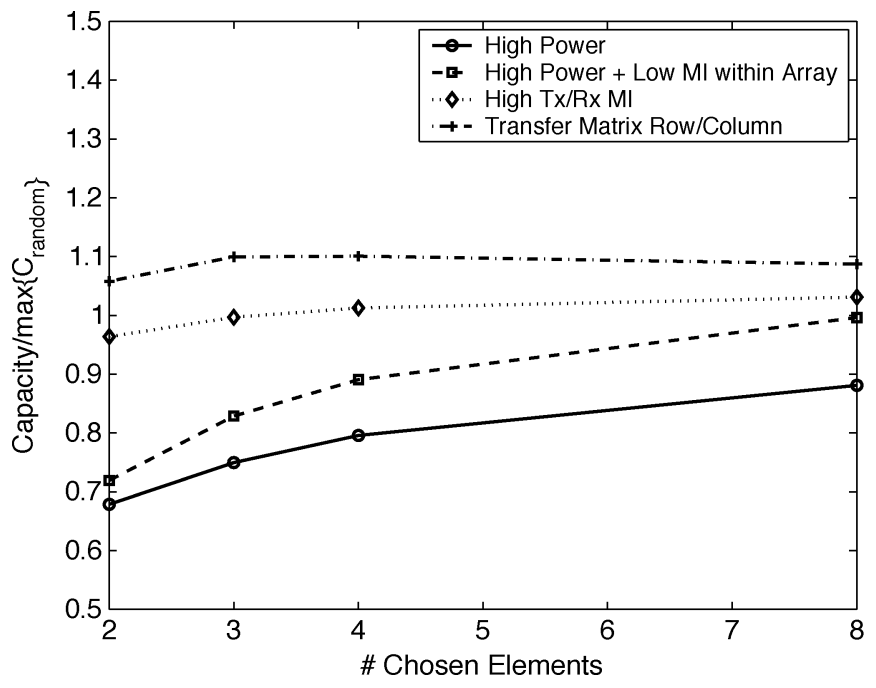

Fig. 5. Selected array capacity normalized by the maximum capacity from 5000 randomly generated arrays versus the number of selected antenna elements. Each point represents an average computed from 150 different channels. The $2 \lambda$ apertures are divided into four gridpoints per wavelength.

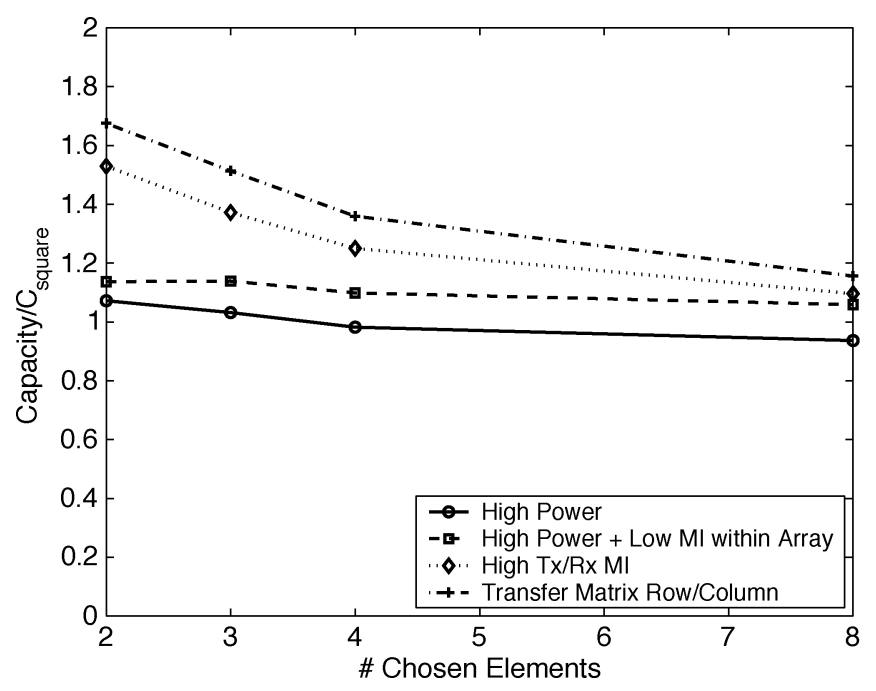

Fig. 6. Selected array capacity normalized by the capacity for the appropriate square array versus the number of selected antenna elements. Each point represents an average computed from 150 different channels. The $2 \lambda$ apertures are divided into four gridpoints per wavelength.

search to identify the maximum capacity is reduced, since more array realizations are needed to cover the search space.

Using this framework, we now explore the performance of the antenna-selection algorithms. As a first computation, the $2 \lambda$ square apertures were divided into square cells at a density of two cells per wavelength (16 transmit and 16 receive array elements). For each channel and array size, the capacity of the array formed by the antenna-selection algorithm was recorded. This capacity was then normalized by the capacity of the random array (of the same size) that yielded the maximum capacity for the channel $\left(\max \left\{\mathrm{C}_{\text {random }}\right\}\right)$ or, alternatively, by the capacity $\mathrm{C}_{\text {square }}$ of the square array in the channel. Additionally, the capacities from the random array computation were formed into a cumulative distribution function (cdf) and the percentile point within the cdf corresponding to the capacity from the algorithmically selected array was recorded. These numbers were then 


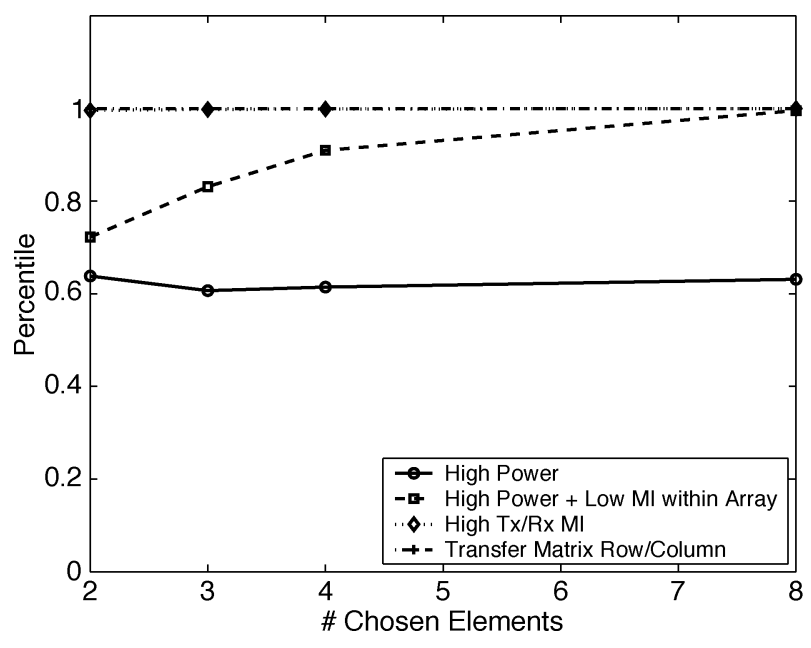

Fig. 7. Percentile performance of the selected array capacity relative to the capacity statistics obtained 5000 randomly generated arrays. Each point represents an average computed from 150 different channels. The $2 \lambda$ apertures are divided into four gridpoints per wavelength.

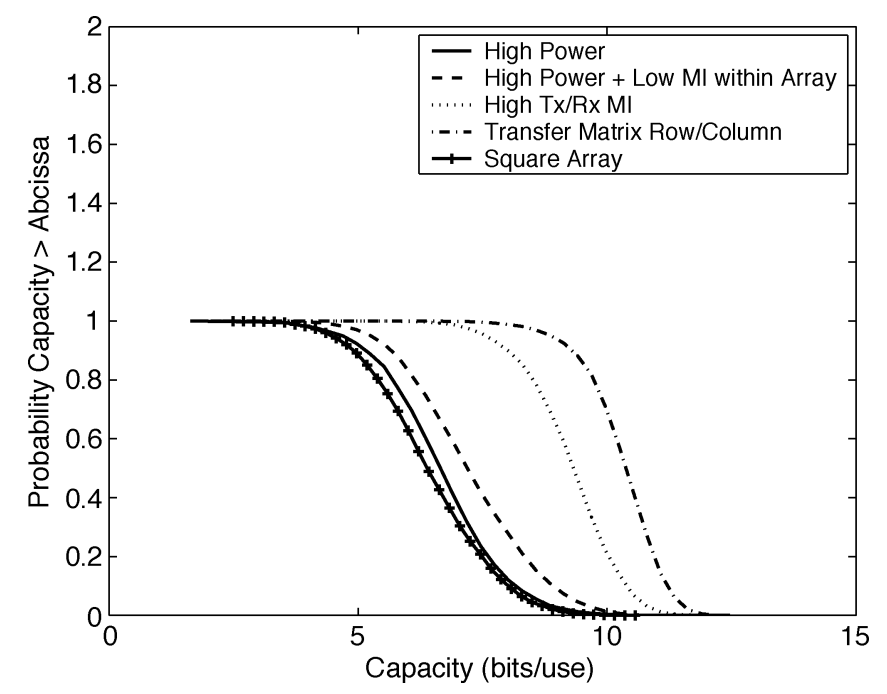

Fig. 8. Complementary cdf of capacity for 5000 randomly generated channels for different two-element arrays selected by the various algorithms compared to the performance of a two-element square array. The $2 \lambda$ apertures are divided into four gridpoints per wavelength.

averaged over the 150 channels for each array size. Figs. 2-4 show the results of these computations. Figs. 5-7 show the same results when four antennas per wavelength were used to discretize the apertures. In each case, the performance obtained when selecting antennas based solely on power (the diagonal elements of the covariance matrix) are included.

These results show that the proposed algorithms outperform selection by power alone. Furthermore, algorithms based on the covariance matrix tend to improve for larger subarrays that more closely mimic the abilities of the full array for which the covariance was originally computed. For such large subarrays, selection based on power alone is a reasonable approach. The performance of the selected arrays relative to that of the square array decreases with increasing array size, since the larger square array is more able to fully exploit the channel spatial properties. This result suggests that using a large fixed array of elements

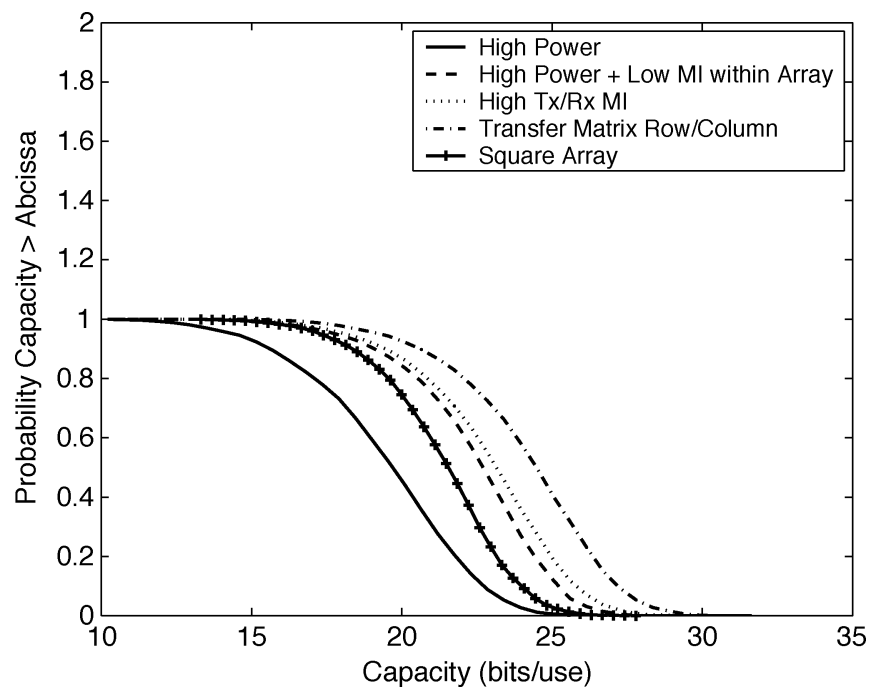

Fig. 9. Complementary cdf of capacity for 5000 randomly generated channels for different eight-element arrays selected by the various algorithms compared to the performance of an eight-element square array. The $2 \lambda$ apertures are divided into four gridpoints per wavelength.

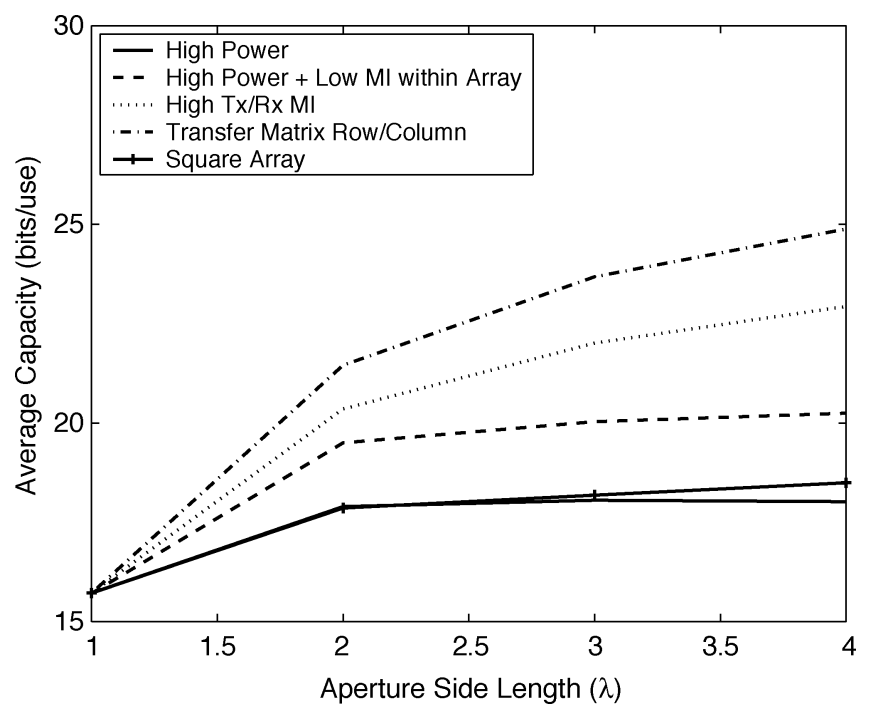

Fig. 10. Average capacity versus aperture size for 5000 randomly generated channels for different four-element arrays selected by the various algorithms compared to the performance of a four-element square array. The $2 \lambda$ apertures are divided into two gridpoints per wavelength.

located on the aperture perimeter is a reasonable approach for achieving high capacity.

Perhaps most striking is the observation that the selection based strictly on the transfer matrix outperforms all other algorithms. This approach is superior since it computes the capacity for each potential arrangement, while the other methods estimate this capacity using the full-array covariance matrix. Selection based on high transmit/receive (Tx/Rx) MI performs nearly as well as the transfer matrix approach, since maximization of this quantity is directly tied to the system capacity. It is noteworthy that although this approach does not perform as well as the best algorithm, it does do better than nearly all (and in most cases all) of the randomly selected arrays, as shown in Figs. 4 and 7. This observation also reinforces the limitations of using a random search to identify the optimal achievable performance. 
The observations discussed previously can be reinforced by applying the antenna selection algorithms to 5000 randomly generated channels. Apertures with side length $2 \lambda$ and discretized with four antennas per wavelength are used. Figs. 8 and 9 show the complementary cdf of capacity for two- and eight-element arrays, respectively, obtained using the different approaches. These results confirm the superiority of selection based upon the transfer matrix. They also demonstrate that, for these circumstances, very reasonable performance can be obtained simply by using a square array around the aperture perimeter, particularly for large array sizes.

Finally, it is interesting to examine the impact of aperture size on the algorithm performance. For this simulation, the capacity averaged from 5000 randomly generated channels is plotted as a function of the square aperture side length. The discretization size is two antennas per wavelength and the selected array size is four elements. Fig. 10 shows the capacity performance. These results confirm the relative performance of the techniques and show that the performance benefit of the best algorithms increases significantly with array size (the ratio of selected to available antennas becomes small).

\section{CONCLUSION}

This paper has presented several algorithms for selecting a subset from a large set of available antennas for MIMO wireless communications. The different algorithms use an iterative approach to provide suboptimal yet good performance with computational efficiency. The performance of the algorithms has been studied using channel matrices obtained from a channel model known to provide good characterization of indoor wireless multipath channels. These results show that simple iterative approaches based upon the large channel matrix or covariance matrices can produce arrays that provide very good capacity. With continued development of large reconfigurable antennas, such algorithms could be used in practice for future generation wireless systems. The results also indicate that, under certain circumstances, placing the array elements around the perimeter of the transmit and receive apertures tends to provide very good performance. This is noteworthy, since implementation of such fixed arrays requires considerably less system complexity.

\section{REFERENCES}

[1] J. H. Winters, "On the capacity of radio communication systems with diversity in a Rayleigh fading environment," IEEE J. Sel. Areas Commun., vol. SAC-5, no. 5, pp. 871-878, Jun. 1987.

[2] G. J. Foschini and M. J. Gans, "On limits of wireless communications in a fading environment when using multiple antennas," Wireless Pers. Commun., vol. 6, pp. 311-335, 1998.

[3] G. G. Raleigh and J. M. Cioffi, "Spatio-temporal coding for wireless communication," IEEE Trans. Commun., vol. 46, no. 3, pp. 357-366, Mar. 1998.

[4] J. W. Wallace and M. A. Jensen, "Intrinsic capacity of the MIMO wireless channel," in Proc. IEEE 56th Vehicular Technology Conf., vol. 2, Vancouver, BC, Canada, Sep. 2002, pp. 701-705.

[5] J. C. Veihl, R. E. Hodges, D. McGrath, and C. Monzon, "Reconfigurable aperture decade bandwidth array," in Proc. IEEE Antennas and Propagation Soc. Int. Symp., vol. 1, Salt Lake City, UT, Jul. 2000, pp. 314-317.

[6] T. Ozdemir, K. F. Sabet, E. Yasan, M. C. Vega, J. L. Ebel, G. L. Creech, C. D. Lesniak, L. P. B. Katehi, and K. Sarabandi, "A hybrid-statistical approach for accurate characterization of MEMS on complex platforms," in Proc. IEEE Antennas and Propagation Soc. Int. Symp., vol. 3, Boston, MA, Jul. 2001, pp. 666-669.

[7] A. F. Molisch, J. H. Winters, and M. Z. Win, "Capacity of MIMO systems with antenna selection," in Proc. IEEE Int. Conf. Communication, vol. 2, Helsinki, Finland, Jun. 2001, pp. 570-574.
[8] D. Gore and A. Paulraj, "Space-time block coding with optimal antenna selection," in Proc. IEEE Int. Conf. Acoustics, Speech, and Signal Processing, vol. 4, Salt Lake City, UT, May 2001, pp. 2441-2444.

[9] D. A. Gore, R. W. Heath, and A. J. Paulraj, "Transmit selection in spatial multiplexing systems," IEEE Commun. Lett., vol. 6, no. 11, pp. 491-493, Nov. 2002

[10] R. W. Heath Jr., S. Sandhu, and A. Paulraj, "Antenna selection for spatial multiplexing systems with linear receivers," IEEE Commun. Lett., vol. 5, no. 4, pp. 142-144, Apr. 2001.

[11] D. A. Gore and A. J. Paulraj, "MIMO antenna subset selection with space-time coding," IEEE Trans. Signal Process., vol. 50, no. 10, pp. 2580-2588, Oct. 2002.

[12] S. Sandhu, R. U. Nabar, D. A. Gore, and A. Paulraj, "Near-optimal selection of transmit antennas for a MIMO channel based on Shannon capacity," in Proc. 34th Asilomar Conf. Signals, Systems, and Computers, vol. 1, Pacific Grove, CA, Oct. 2000, pp. 567-571.

[13] A. Gorokhov, "Antenna selection algorithms for MEA transmission systems," in Proc. IEEE Int. Conf. Acoustics, Speech, and Signal Processing, vol. 3, Orlando, FL, May 2002, pp. 2857-2860.

[14] M. A. Khalighi, J. Brossier, G. Jourdain, and K. Raoof, "Water filling capacity of Rayleigh MIMO channels," in Proc. IEEE 12th Int. Symp. Personal, Indoor, and Mobile Radio Communications, vol. 1, San Diego, CA, Sep. 2001, pp. 155-158.

[15] E. Baccarelli and M. Biagi, "Power-allocation policy and optimized design of multiple-antenna systems with imperfect channel estimation," IEEE Trans. Veh. Technol., vol. 53, no. 1, pp. 136-145, Jan. 2004.

[16] T. M. Cover and J. A. Thomas, Elements of Information Theory. New York: Wiley, 1991.

[17] K. Mardia, J. Kent, and J. Bibby, Multivariate Analysis. New York: Academic, 1980.

[18] T. K. Moon and W. C. Stirling, Mathematical Methods and Algorithms for Signal Processing. Englewood Cliffs, NJ: Prentice-Hall, 2000.

[19] J. W. Wallace and M. A. Jensen, "Modeling the indoor MIMO wireless channel," IEEE Trans. Antennas Propag., vol. 50, no. 5, pp. 591-599, May 2002.

[20] A. A. M. Saleh and R. A. Valenzuela, "A statistical model for indoor multipath propagation," IEEE J. Sel. Areas Commun., vol. SAC-5, no. 2, pp. 128-137, Feb. 1987.

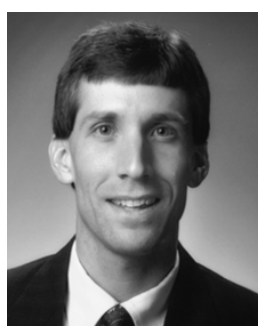

Michael A. Jensen (S'93-M'95-SM'01) received the B.S. (summa cum laude) and M.S. degrees in electrical engineering from Brigham Young University (BYU), Provo, UT, in 1990 and 1991, respectively, and the Ph.D. in electrical engineering from the University of California, Los Angeles (UCLA) in 1994.

From 1989 to 1991, he was a Graduate Research Assistant in the Lasers and Optics Laboratory, BYU. From 1991 to 1994 , he was a Graduate Student Researcher in the Antenna Laboratory, UCLA. Since 1994, he has been with the Electrical and Computer Engineering Department, BYU, where he currently is an Associate Professor. His main research interests include antennas and propagation for personal communications, microwave circuit design, radar remote sensing, numerical electromagnetics, and optical fiber communications.

Dr. Jensen is a Member of Eta Kappa Nu and Tau Beta Pi. He currently serves on the Joint Meetings Committee, IEEE Antennas and Propagation Society, and has served as the Technical Program Chair, 2000 IEEE International Symposium on Antennas and Propagation, and as Symposium Vice Chair for the 2003 IEEE Topical Conference on Wireless Communications Technology. He received a National Science Foundation Graduate Fellowship in 1990. He was awarded the H. A. Wheeler Paper Award by the IEEE TRANSACTIONS ON ANTENNAS AND PROPAGATION in 2002 and the Best Paper Award at the 1994 IEEE International Symposium on Antennas and Propagation.

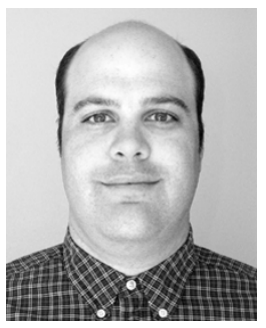

Matthew L. Morris received the B.S. (magna cum laude) degree in physics from Brigham Young University (BYU), Provo, UT, in 2000, where he is currently working toward the Ph.D. degree in electrical and computer engineering.

Since 2000, he has been a Graduate Research Assistant in the Wireless Communications Laboratory, BYU. His reseach is concentrated in the area of multiple-input-multiple-output (MIMO) antenna systems. 